\title{
EXPLORATION OF UNDERGROUND MECHANICAL ENVIRONMENT MONITORING SYSTEM ACCORDING TO ZIGBEE WIRELESS COMMUNICATION
}

\author{
Xuhong $\mathrm{Li}^{1}$, Tao Xu ${ }^{1}$, Quoc Tien $\mathrm{Le}^{2}$ \\ ${ }^{1}$ School of information science and Engineering, Zaozhuang University, Shandong China \\ ${ }^{2}$ Haiphong Department of Education and Training, Haiphong, Vietnam \\ Email: taoxu4912@126.com
}

\begin{abstract}
This study aims to better realize the application of wireless communication technology in the underground mechanical environment monitoring system. In this research, we proposed solution to the problems of high power consumption and complicated construction in wired detection. Firstly, the technical characteristics of zigbee wireless communication are briefly analyzed, and then the hardware and software parts of underground mechanical environment monitoring system are designed; including the processor of the system, zigbee wireless communication module, driver module, and sensor module. Based on outcomes, the multidimension signal monitoring design under the mechanical environment is carried out by means of multi-dimension empirical mode method. Finally, by simulating the underground mechanical experiment environment, the performance of the monitoring system is evaluated. The results show that the temperature and humidity detected by the environmental monitoring system are close to the actual temperature and humidity, the microcontroller can also show the flame conditions in the experimental environment in time, and the multi-dimensional signal amplitude of the underground environment is healthy. Therefore, it can be concluded that the environmental monitoring system designed based on zigbee wireless communication technology can accurately and timely monitor environmental changes, which not only has a wide range of social application value but also plays a decisive role in promoting the development of related fields.
\end{abstract}

Keywords: Zigbee Technology, Mechanical Environment Monitoring System, Protocol Conversion, Multivariate Empirical Mode Method, Low Difference Sequence Sampling Method.

\section{Introduction}

With the sustained development of the national economy, the utilization of coal resources in China has promoted by the continuous enhancement of the country's massive industry level [1]. Nowadays, with the increasing demand for coal, the mining technology of coal industry is continuously updated and upgraded. China generally adopts underground mining for coal, and risk events in the mining environment severely restrict the safety of production [2]. For example, coal dust pollution and high gas concentration generated during underground mining threaten workers' safety all the time. Therefore, to avoid coal mine operation accidents, it is necessary to conduct real-time monitoring of underground operation environment, personnel status, and equipment status [3].

The traditional underground mechanical environmental monitoring system mostly adopts the wired method to transmit the underground mechanical environmental data, which has many limitations. In addition to redundant wiring and high work intensity, it can't meet the continually changing requirements of underground road structure, which also leads to the increase of monitoring cost [4]. The position of the monitoring system is fixed, and it is comfortable to appear in blind areas, so it is challenging to achieve all-round underground monitoring [5]. With the progress of wireless technology and integration, the emergence of highperformance radio frequency chips lays a foundation for the upgrade of the underground mechanical environment monitoring system [6]. With the development of wireless network technology, the data transmission mode of all walks of life adopts this new communication mode, which has been widely used in military, geological, financial, and transportation fields [7, 8]. In recent years, China has also gradually used advanced technologies such as wireless ad hoc networks and intelligent sensing in underground safety monitoring and early warning to form a wireless underground safety and rescue system. For example, the wireless communication 
system based on rescue of mine accident significantly improves the emergency communication range and command debugging of the underground rescue process, to further guarantee the safety of underground workers [9]. Among wireless network technologies, bluetooth, Wi-Fi, and zigbee are the most widely used technologies, but they all have their disadvantages and advantages, so they can't be generalized. For underground machinery environmental monitoring systems, with their own advantages, combined with protocol conversion technology, the application range of wireless networks can be enhanced through multiple network integration methods.

In short, to better apply wireless communication technology for monitoring of the underground mechanical environment, combined study with protocol conversion technology and based on zigbee wireless communication technology is evaluated. The monitoring system is designed for the underground mechanical environment, trying to solve the monitoring problems faced by the wired monitoring system and vigorously develop the wireless information transmission mode.

\section{Methods}

\subsection{Analysis of zigbee wireless communication technology}

Zigbee wireless communication technology has properties of clear structure, small power consumption, low speed, and short transmission distance. Therefore, this communication technology is mainly applicable to fields requiring automatic control and sensing, remote control and positioning, and it can also act as a combined facility in communication equipment. The communication protocol involved starts from the physical layer, passes through the media access stage, then enters the transmission stage, and finally realizes the work of the whole process through the network stage and the application stage [10-12]. The advantages of zigbee wireless communication technology are shown in table 1.

Table 1. Advantages of zigbee wireless communication technology

\begin{tabular}{|c|c|}
\hline Main features & Specific performance \\
\hline $\begin{array}{l}\text { Relatively low } \\
\text { delivery rate }\end{array}$ & $\begin{array}{l}\text { The transmission rate of underground environmental monitoring data is not required to } \\
\text { be high, while zigbee wireless communication technology is a transmission mode with } \\
\text { low rate. Therefore, zigbee wireless communication technology can meet the } \\
\text { requirements of underground environmental monitoring. }\end{array}$ \\
\hline $\begin{array}{l}\text { Low power } \\
\text { consumption }\end{array}$ & $\begin{array}{l}\text { The wireless information transmission system needs to be powered by batteries. Due to } \\
\text { the low transmission rate of zigbee, the minimum power of transmitting information is } \\
\text { only } 1 \mathrm{~mW} \text {, and it can be directly put into hibernation in short delay and idle state. At this } \\
\text { time, the power consumption of the system is only } 30 \mathrm{w} \text {. Wireless communication nodes } \\
\text { consume very little power. }\end{array}$ \\
\hline $\begin{array}{l}\text { Prompt } \\
\text { response }\end{array}$ & $\begin{array}{l}\text { Zigbee wireless communication technology has a short time delay interval and is } \\
\text { activated from hibernation very quickly. The delay of the technology is } 30 \mathrm{~ms} \text { for the } \\
\text { search device, and the latency of the system's access channel and the latency of sleep } \\
\text { activation are both } 15 \mathrm{~ms} \text {. }\end{array}$ \\
\hline Cost saving & $\begin{array}{l}\text { Compared with bluetooth, WiFi, and other technologies, zigbee wireless communication } \\
\text { technology has a simple protocol, low transmission rate, and low power consumption, } \\
\text { thus significantly saving costs and providing possibilities for large-scale network layout } \\
\text { [13]. }\end{array}$ \\
\hline $\begin{array}{l}\text { Long distance } \\
\text { conveyance }\end{array}$ & $\begin{array}{l}\text { Environment roadway of underground operation is very long, and communication } \\
\text { transmission distance should be more than } 10 \mathrm{~km} \text {. The distance between the } \\
\text { communication nodes of the zigbee wireless communication technology can reach } 3 \mathrm{~km} \text { by } \\
\text { increasing the transmission power, and the relay path can be used to make the } \\
\text { transmission distance farther to meet the actual needs. }\end{array}$ \\
\hline Reliability & $\begin{array}{l}\text { Zigbee itself has an anti-interference direct sequence spread spectrum communication } \\
\text { technology, so all signals without spread spectrum factor will be filtered out to ensure } \\
\text { that useful signals are read and received. }\end{array}$ \\
\hline $\begin{array}{l}\text { Many nodes } \\
\text { which are } \\
\text { accommodated }\end{array}$ & $\begin{array}{l}\text { Each zigbee network can accommodate more than } 65,000 \text { nodes, forming a huge network } \\
\text { system. }\end{array}$ \\
\hline Safe & $\begin{array}{l}\text { Zigbee wireless communication technology provides the function of data cyclic } \\
\text { redundancy check, which can't be used to analyze the original signal without } \\
\text { authorization. }\end{array}$ \\
\hline $\begin{array}{l}\text { Automatic } \\
\text { monitoring }\end{array}$ & $\begin{array}{l}\text { When the zigbee communication node runs out of energy or has problems, it will } \\
\text { automatically quit, thus adding new nodes. }\end{array}$ \\
\hline
\end{tabular}


The zigbee wireless communication technology in this research is embodied in the form of network, and the relay node can realize multi-level jump to realize long-distance data transmission. Most of the relay nodes in the system have routing functions. Compared with the tree network structure, a relay node in the network structure can be connected with the nearby relay nodes, so it has more reliability and security $[14,15]$.

\subsection{Design of hardware and software of underground mechanical environment monitoring system}

Hardware design of underground mechanical environment monitoring system is the basic condition for the system to run. The module includes the processor, zigbee wireless communication module, driver module, and sensor module. The sensor module also includes the following parts: air, fire, humidity, and wind. The node connection between each module is shown in figure 1 .

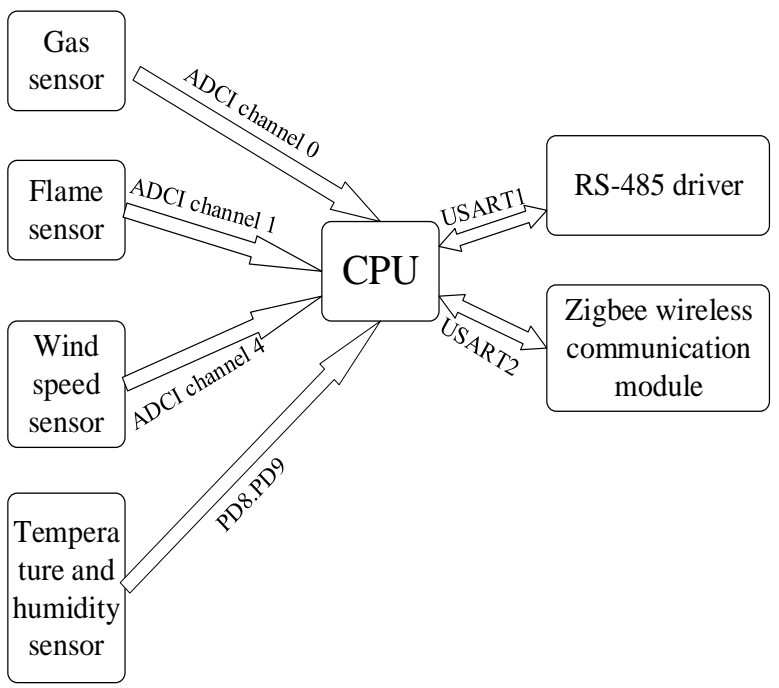

Figure 1: Hardware module connection diagram of the monitoring system

In the hardware design of underground mechanical environment monitoring system, zigbee wireless communication module can be developed in two ways. One method is to purchase chips and then design their peripheral circuits. The other way is to maintain the stability of system performance by purchasing off-the-shelf zigbee modules. In this research, it chooses the second one.

First, it is difficult to ensure the security and stability of wireless communication by avoiding the designed protocol stack line and peripheral circuit. Second, it reduces a lot of time cost. The zigbee wireless communication module in this research is shown in figure 2.

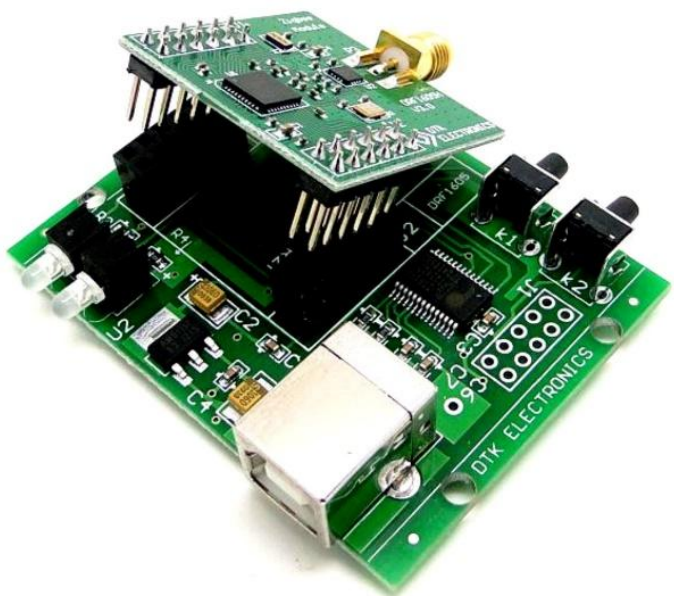

Figure 2: Physical picture of zigbee wireless communication module

The zigbee module of H7710E GPRS DTU adopts an embedded design, and its transmission distance reaches $10 \mathrm{~m}$ to $100 \mathrm{~m}$. Due to the increased transmission power consumption of this module, its maximum visual transmission distance can reach about $2000 \mathrm{~m}$. The $2.4 \mathrm{GDSSS}$ spread spectrum technology of this module greatly enhances the antiinterference capability of nodes. Its center node, routing node, and terminal node can be designed at will, and the network type can be designed according to the needs of several different network forms, including star network, tree network, mash network, chain network, and so on [16-18].

The software design of underground mechanical environment monitoring system includes four sensors of monitoring nodes, namely flame sensor, humidity sensor, wind speed sensor, and gas sensor. In the design of the flame sensor, five sensors are used to output the analog digital signal. The corresponding azimuth is different, and the real-time flame situation can be detected from different angles. The corresponding clock line ports of the humidity sensor are push-pull output for enhanced driving capability and floating input.

The rotor blades of the wind speed sensor are blown by the wind and are rotated to the alternator to generate electricity. The voltage signal output by the motor at this time is called an alternating current signal; its purpose is to facilitate subsequent information collection.

In this research, by designing the circuit of rectifier filter, the alternating current signal is transformed into a stable direct current signal through transmission. However, the direct current signal in actual operation is not necessarily completely stable, and occasionally, there is a small fluctuation.

Therefore, to obtain accurate information of the direct current signal, it is necessary to continuously collect at least 5 times of simulated signals when the query command is given by the pull-up machine 
position method, and then obtain the voltage signal corresponding to the detected voltage and wind speed. In this way, the detected voltage is converted into the wind speed by 3.5 times of the signal. The gas sensor is digitally simulated in a continuous approximation mode, which can measure the signal source outside and inside of 15 channels. The individual channels can be converted in a single but continuous scan mode. The analog input signals applied to this system are respectively the output signals of the gas sensor, the output signals of the flame sensor, and the output signals of the wind speed sensor. The three analog signals are input together and initialized.

The data transmission volume of underground mechanical environment monitoring system is large and the transmission rate is fast. Therefore, the communication protocol of this system adopts the Modbus protocol format.
The implementation of this protocol divides its nodes into three types, namely the host computer, the slave computer, and the detection node. The detection node contains both the routing node and the terminal node, and the routing node is automatically completed in the process of information transmission. The sequence of program flow of the host is to send and query instructions in order by means of polling. After waiting for a monitoring node to send the instructions, it can receive the normal response data frame. At this point, it means that the monitoring node is running normally and the data displayed on the system interface is read at the same time. If the returned data frame is abnormal, the communication error is indicated, and the error information needs to be recorded before entering the query of the next node. The process implementation of the host program is shown in figure 3 .

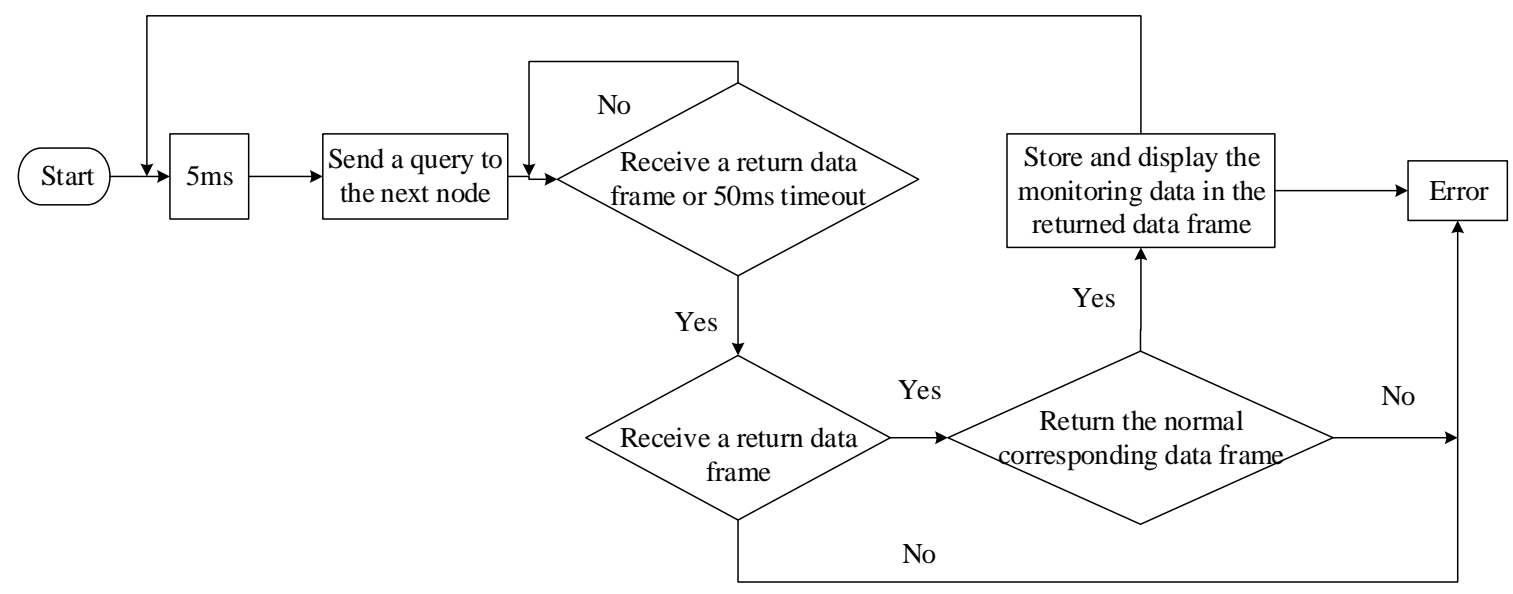

Figure 3: Process realization diagram of the host program

The main function of the slave machine is to forward the monitoring node corresponding to the query instructions on the host computer and forward the returned data frame of the monitoring node to the host computer. The receiving query instruction flow of the slave machine is shown in figure 4 .

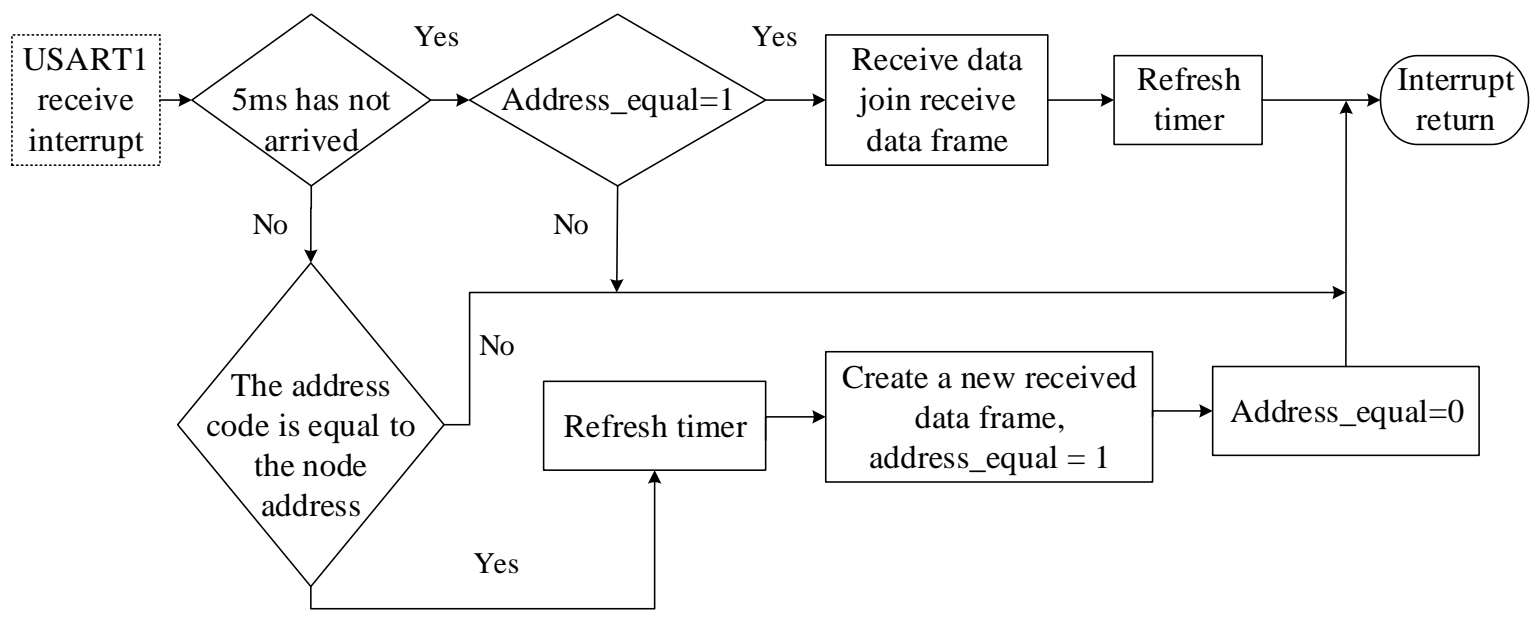

Figure 4: Flow chart of receiving query instruction from the slave 
The $5 \mathrm{~ms}$ setting in timer indicates that a data frame is completed and needs to be processed in a timed interrupt. It takes a long time to execute the query instruction, which usually includes CRC check, monitoring environment information to return the monitoring data, or forwarding the query instruction. However, the time it takes to execute the operation process is difficult to meet the requirements and principles of short-term completion, so the solution is to change the value in the interrupt, and then the main program can query the variable value to complete the corresponding operation.

After adjusting the time to the state of 1 and stopping the timing of timer, it means timing to $5 \mathrm{~ms}$. Then, the pointer of the received data frame buffer is pointed to the starting position to ensure that the next byte received is in front of the newly received data frame. At this time, the flag of executing the query instruction or forwarding the query instruction is set to 1 .

\subsection{Design of multidimensional signal monitoring in mechanical environment}

The downhole mechanical environment is complicated, multi-channel signals can't be analyzed synchronously, and the disunity of decomposition scale may lead to signal disorder. Besides, the coupling and redundancy phenomena between signals tend to cause problems such as modal alignment in the data analysis stage. Therefore, the method of multivariate empirical mode decomposition is used to decompose the monitoring data so as better to monitor the vibration signals in the mechanical environment.

First, the sequence of vector set of a threedimensional signal $\{v(\mathrm{t})\}_{t}^{T}=\left\{v_{1}(\mathrm{t}), v_{2}(\mathrm{t}), v_{3}(\mathrm{t})\right\}$ and the vector $X^{n}=\left\{x_{1}^{n}, x_{2}^{n}, x_{3}^{n}\right\}$ in $n$th direction is set. Where, $\mathrm{T}$ represents the length of the signal. A set of uniformly distributed direction vector $\mathrm{X}$ is obtained by low-difference sequence sampling method. The signal group $v(\mathrm{t})$ is then extended to the projection of the vector $X^{n}$ in $n$th direction.

Therefore, the corresponding moments $t_{i}^{n}$ of the maximum and minimum values of the projection function are found, $i=1,2,3, \ldots, n$, where $n$ is the number of the most valued points, and all envelope functions corresponding to the vector in direction

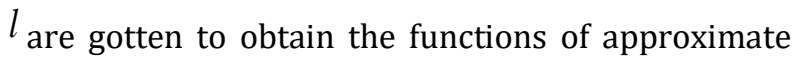
values.

$$
M(\mathrm{t})=\frac{1}{l} \sum_{n=1}^{l} E^{n}(\mathrm{t})
$$

Finally, the intrinsic modal function $h(t)=\mathrm{V}(t)-M(t)$ is extracted, and the screening process of the multiple empirical modal decomposition method is performed. The above process is repeated to decompose the original signal data, which can be expressed as equation (2).

$$
\{v(\mathrm{t})\}_{t}^{T}=\sum_{i=1}^{d} h_{i}(\mathrm{t})+r(\mathrm{t})
$$

Where, $d$ is the number of extra multiple layers decomposed, $h(\mathrm{t}) \quad$ is $h(\mathrm{t})\left\{h_{i}^{1}(\mathrm{t}), h_{i}^{2}(\mathrm{t}), h_{i}^{3}(\mathrm{t})\right\}_{t=1}^{T}$, and $r(\mathrm{t})$ is $\mathrm{r}(\mathrm{t})\left\{r_{i}^{1}(\mathrm{t}), r_{i}^{2}(\mathrm{t}), r_{i}^{3}(\mathrm{t})\right\}_{t=1}^{T}$.

Through the above analysis process, multichannel signal decomposition can be realized, which is very suitable for the monitoring of vibration signals in the underground mechanical environment.

\section{Results and Discussion}

\subsection{Temperature / humidity test of underground mechanical environment monitoring system}

The test platform of underground mechanical environmental monitoring system is built to verify whether the system designed based on zigbee wireless communication can accurately monitor underground environmental information.

In this research, monitoring data of temperature and humidity at different time points under the monitoring system are recorded through testing, as shown in figure 5.

The experimental simulation environment in this research is an indoor roadway with a similar underground environment, and a monitoring node is set every 30 meters, with a total of 3 nodes. A node is arranged at the corner of the corridor, which is used as the middle node for experimental verification. However, it can be observed from figure 5, the temperature and humidity measured by the underground mechanical environmental monitoring system at different time points are not significantly different from the actual temperature and humidity, and the values are relatively close. 


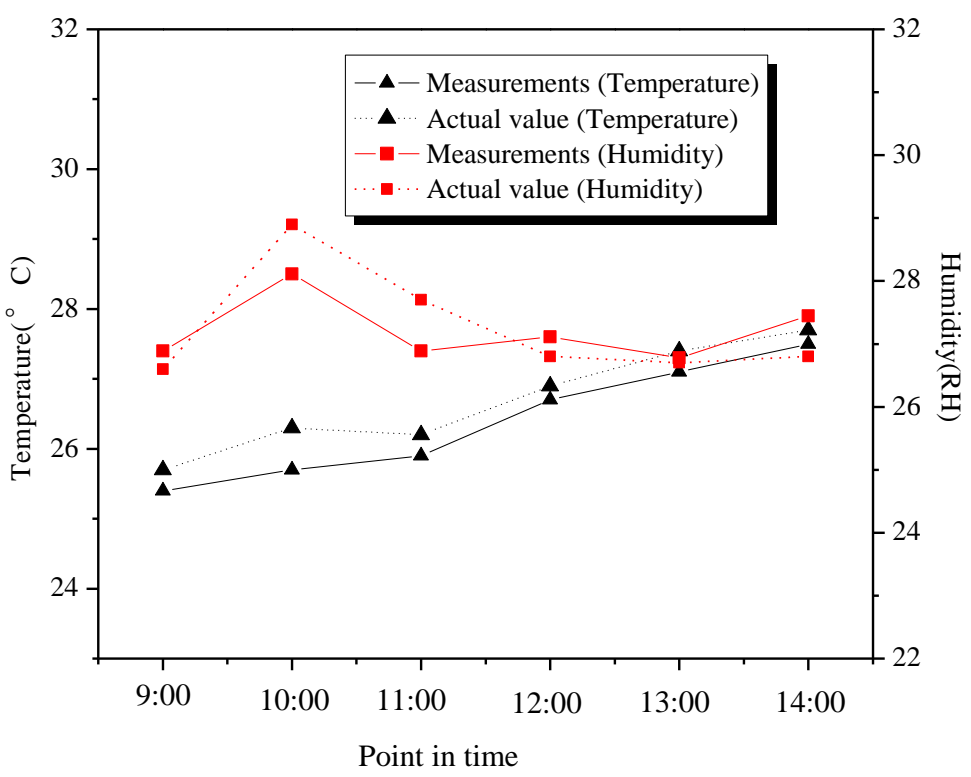

Figure 5: Comparison of measured temperature and humidity and actual temperature and humidity at different time points

This result from figure 5 indicating that the underground monitoring system designed in this research has good stability and can detect changes in environmental information relatively accurately.

In this research, the gas in the lighter is measured with a gas sensor. The fuel used by the lighter is mainly a flammable gas with a state similar to that of the downhole ambient gas. The lighter is close to the sensor, when the lighter is opened the gas inside is sprayed out.

The artificially changed flame distance is used for live monitoring, and the corresponding voltage values of different flame distances are tested, as shown in figure 6 .

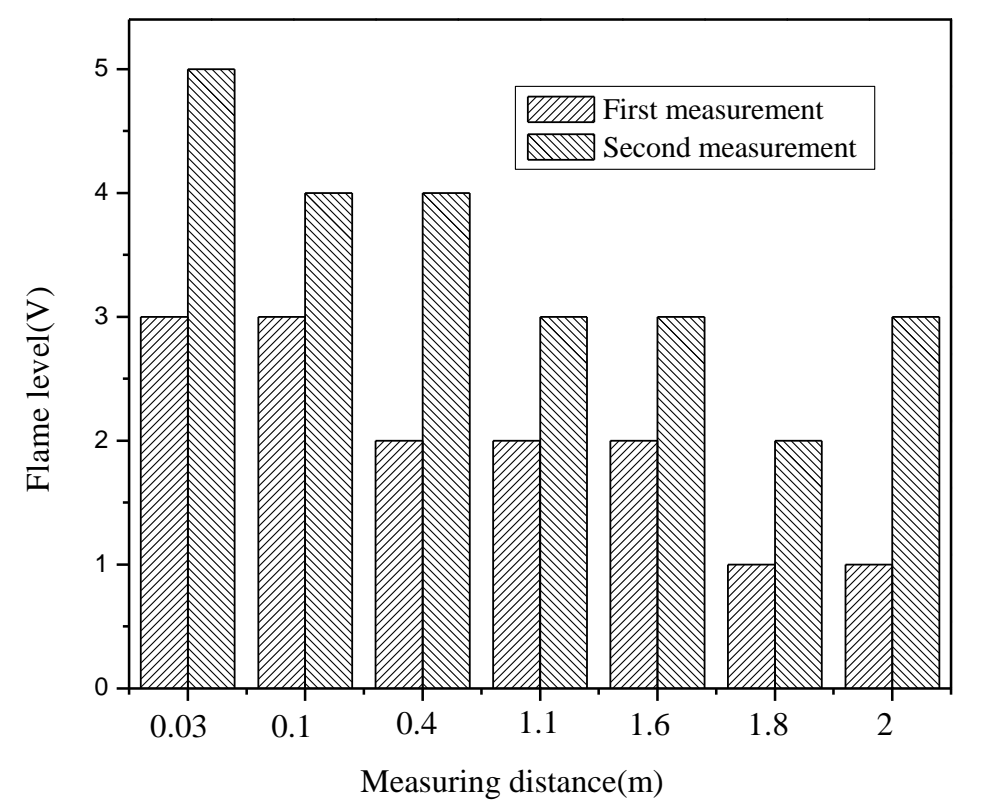

Figure 6: Corresponding voltage values at different flame distances

It can be observed from the above figure, when the system is close to the flame sensor, the higher the output voltage. At this time, the microcontroller can display the current flame situation. When the surrounding environment changes, the underground mechanical environment monitoring system can timely and accurately reflect the environmental information to meet the actual underground environment monitoring needs. In the experiment, not only the monitoring data can be displayed in real time from the host, but also the stored historical data can be consulted at any time. This property enables the system designed in this research to allow people to master the environmental information of 
underground machinery in real time, to achieve optimal scheduling management, and to provide guidance for the formulation of effective rescue plans in case of accidents.

\subsection{Monitoring and evaluation of underground mechanical environment vibration signal}

Figure 7 and figure 8 are the signal spectrum diagrams of downhole environmental vibration at $6 \mathrm{~Hz}$ and $8 \mathrm{~Hz}$, respectively.
It can be observed from them that the amplitude of downhole machinery at $6 \mathrm{~Hz}$ is more dispersed than that at $8 \mathrm{~Hz}$. With the increase of the rotating speed, the amplitude also increases obviously, continuously experiencing a process from high to low, from low to high.

In both cases, the peak value appears, the vibration phase keeps changing, and the vibration amplitude is pulled back to a smaller level from a higher level, indicating that the mechanical environment is running normally at this time, thus verifying the feasibility of the monitoring system.

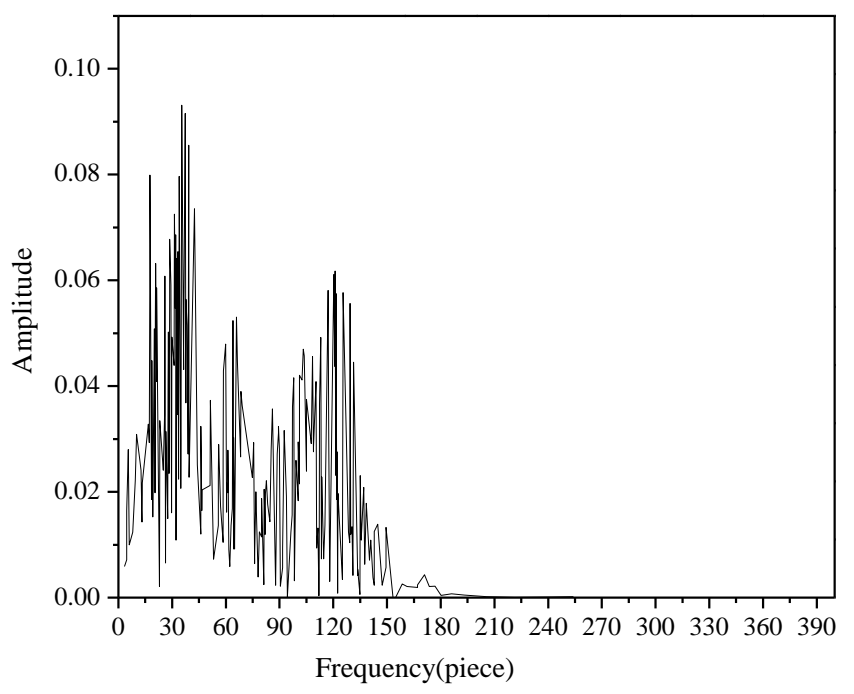

Figure 7: Vibration spectrum of underground mechanical environment at $6 \mathrm{~Hz}$

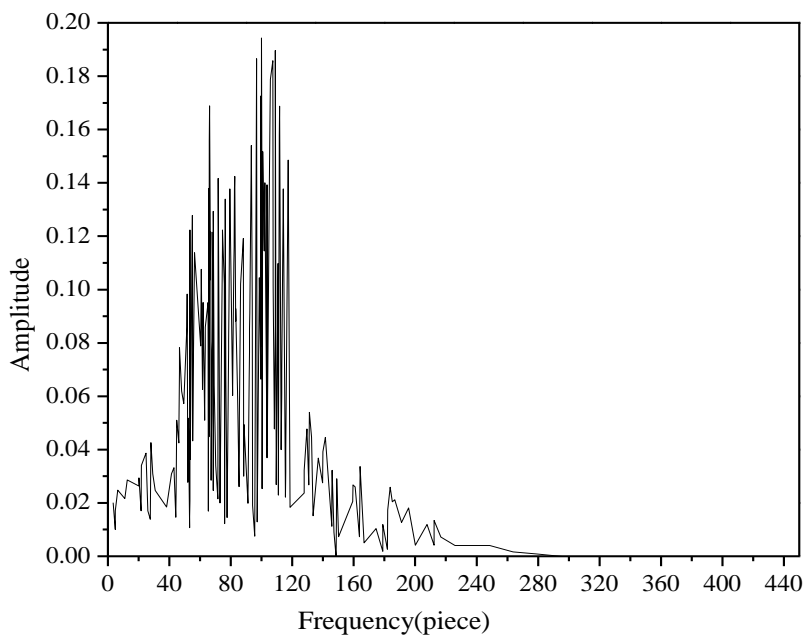

Figure 8: Vibration spectrum of underground mechanical environment at $8 \mathrm{~Hz}$

To sum up, the monitoring system based on zigbee wireless communication can be accurate to the real-time monitoring of downhole mechanical environment information, and its operation is stable and reliable.

Therefore, it can be known that the program designed in this research realizes the process of monitoring the mechanical environment information of the node and follows the communication process of the protocol, so as better to play the role of zigbee wireless communication technology and meet the requirements of underground mechanical environment monitoring. 


\section{Conclusions}

To improve the performance of underground mechanical environmental monitoring system, in this study, the system is designed by virtue of the advantages of zigbee wireless communication technology. The underground mechanical environmental monitoring system developed from the hardware and software modules of the monitoring system, and simulation of environmental information is conducted for the designed system. The results show that the environmental monitoring system studied in this research is accurate in both the measurement of temperature and humidity and the evaluation of underground mechanical environmental vibration signals. When the flame distance is artificially changed to measure the voltage value, the system can reflect the changes in the monitored environment information in time, thus realizing the remote monitoring of the underground environment.

The underground environment monitoring system designed in this research can achieve the expected purpose. Due to the limited experimental resources, although the test environment of this experiment is shielded from interference, the actual underground environment is quite complicated with many interference factors. Therefore, in the following experimental research, continuous improvement is needed to improve the antiinterference ability of the system.

\section{References}

[1] Belle B, Cliff D. Improved TARP development based upon mine specific data. International Journal of Mining Science and Technology, 2018, 28(3), pp. 477-481.

[2] Almeida Oliveira T, Godoy E P. Zigbee wireless dynamic sensor networks, feasibility analysis and implementation guide. IEEE Sensors Journal, 2016, 16(11), pp. 4614-4621.

[3] Gao M, Wang P, Wang Y, et al. Self-powered ZigBee wireless sensor nodes for railway condition monitoring. IEEE Transactions on Intelligent Transportation Systems, 2017, 19(3), pp. 900-909.

[4] Chen K L, Chen Y R, Tsai Y P, et al. A novel wireless multifunctional electronic current transformer based on ZigBee-based communication. IEEE Transactions on Smart Grid, 2016, 8(4), pp. 1888-1897.

[5] S.K. Gugulothu, N. Prabhu Kishore, V. Phani Babu, Girish Sapre (2019). Cfd Analysis on Different Piston Bowl Geometries by Using Split Injection Techniques. Acta Mechanica Malaysia, 2(1): 23-28.
[6] Kadhum Audaa Jehhef and Mohamed Abed Al Abas Siba (2019). Effect Of Surfactant Addition On The Nanofluids Properties: A Review. Acta Mechanica Malaysia, 2(2): 1-19, DOI: 10.26480/amm.02.2019.01.19

[7] Om Prakash Singh, Gaurav Kumar, Mukesh Kumar (2019). Role of Taguchi And Grey Relational Method in Optimization of Machining Parameters of Different Materials: A Review. Acta Electronica Malaysia, 3(1): 19-22.

[8] Farah Ruzanna Ridzuan, Hairulnizam Mahdin, Shahreen Kasim, Mohd Sanusi Azmi (2019). An Image-Based Captcha System Using Click. Acta Electronica Malaysia, 3(1): 23-25.

[9] Akbar Zada, Hafiz Ullah (2019). Decomposition of $\mathrm{Cm}$ Through Q-Periodic Discrete Evolution Family. Matrix Science Mathematic, 3(1): 09-12.

[10] Sharma M, Maity T. Low cost low power smart helmet for real-time remote underground mine environment monitoring. Wireless Personal Communications, 2018, 102(1), pp. 149-162.

[11] Moridi M A, Sharifzadeh M, Kawamura Y, et al. Development of wireless sensor networks for underground communication and monitoring systems (the cases of underground mine environments). Tunnelling and Underground Space Technology, 2018, 73, pp. 127-138.

[12] Plakhotnij S A, Klyuchko O M, Krotinova M V. Information support for automatic industrial environment monitoring systems. Electronics and control systems, 2016, (1), pp. 29-34.

[13] Kim S Y, Yu J H, Yu Y G, et al. Database enhancement for development of open-pit mine monitoring system in open source environments. Journal of the Korean Society of Surveying, Geodesy, Photogrammetry and Cartography, 2016, 34(1), pp. 21-32.

[14] Akkaş M A. Using wireless underground sensor networks for mine and miner safety. Wireless Networks, 2018, 24(1), pp. 17-26.

[15] Qiu B. Design of mobile 4G gateway based on zigbee wireless sensor network. International Journal of Online and Biomedical Engineering, 2018, 14(11), pp. 117-132.

[16] Kim S H, Chong P K, Kim T. Performance study of routing protocols in ZigBee wireless mesh networks. Wireless Personal Communications, 2017, 95(2), pp. 1829-1853.

[17] Mathurkar S S, Thakare S S. Design and implementation of coal mine physiological parameters monitoring protocol. International Journal on Recent and Innovation Trends in Computing and Communication, 2017, 5(1), pp. 125-130.

[18] Sangeetha M, Susila M M. Advanced paddy harvest monitoring with zigbee system. International Journal of Pure and Applied Mathematics, 2018, 119(7), pp. 1987-1993. 